\title{
Microwave radiative transfer intercomparison study for 3-D dichroic media
}

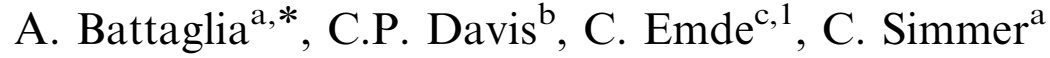 \\ ${ }^{\text {a } M e t e o r o l o g i c a l ~ I n s t i t u t e, ~ U n i v e r s i t y ~ o f ~ B o n n, ~ A u f ~ d e m ~ H u g e l, ~ 20, ~} 53121$ Bonn, Germany \\ ${ }^{\mathrm{b}}$ School of Geosciences, University of Edinburgh, Edinburgh EH9 3JN, UK \\ ${ }^{\mathrm{c}}$ Deutsches Zentrum fuer Luft- und Raumfahrt (DLR) Oberpfaffenhofen, 82234 Wessling, Germany
}

Received 30 August 2006; accepted 26 September 2006

\begin{abstract}
Three different numerical methods capable of solving the radiative transfer of microwave radiation within 3-D dichroic media are compared. A case study, represented by an intense rain shaft populated by perfectly oriented oblate raindrops, is analysed in detail, including a discussion of the behaviour of all four Stokes components.

Results demonstrate an acceptable agreement between all Monte Carlo methods. The method based on a discrete ordinates scheme agrees only qualitatively with the Monte Carlo outputs. Because of its lower computational cost the backward Monte Carlo technique based on importance sampling represents the most efficient way to face passive microwave radiative transfer problems related to optically thick 3-D structured clouds including non-spherical preferentially oriented hydrometeors.
\end{abstract}

(C) 2006 Elsevier Ltd. All rights reserved.

Keywords: Microwave radiative transfer; 3-Deffects; Dichroic media; Polarization

\section{Introduction}

The interaction of microwave radiation with clouds and precipitation has been studied for decades. The physical process is adequately described by the vector radiative transfer equation ( $V R T E$ hereafter) (for details see [1,2]), that can be solved with many different methodologies, a review of which is provided by Mätzler [3]. Only very recently techniques have been developed to numerically solve the radiative transfer equation for the full Stokes vector in 3-D environment in the presence of non-isotropic media [4-7]. The latter condition frequently occurs in nature because large atmospheric hydrometeors like falling raindrops, snow and other ice crystals, tend to have non-spherical shapes and prefer horizontal orientations. Thus falling precipitation represents a dichroic medium and polarization effects can play an important role. Moreover the combination of scattering effects, the large spatial variation of the precipitating hydrometeors in the atmosphere (compared to sensor footprints) and the partly reflecting properties of the surface puts weight on the consideration of

\footnotetext{
*Corresponding author. Tel.: + 49228735779 ; fax: + 49228735188.

E-mail address: batta@uni-bonn.de (A. Battaglia).

${ }^{1}$ Formerly at University of Bremen, 28359 Germany.
} 
three dimensional effects in microwave radiative transfer as well (for an overview of 3-D issues in the $M W$ region see [8]).

Although many benchmark results are available for RT within multilayered 1-D media (see [9-12]) or 3-D $[4,7,13-16]$, up to now there are no benchmark results for 3-D scenes in presence of dichroic material, and no intercomparison studies have been carried out among codes that have these capabilities.

In this work, we consider a single 3-D scenario involving non-spherical hydrometeors perfectly oriented in the horizontal plane. The $R T$ computations in the $M W$ region are performed by different models and the outputs are compared and discussed.

\section{Methods to solve the vector radiative transfer equation}

The VRTE for monochromatic or quasi-monochromatic radiative transfer is generally written in its integrodifferential form as:

$$
\frac{\mathrm{d} \mathbf{I}(\vec{r}, \hat{s})}{\mathrm{d} r}=-\langle\mathbf{K}(\vec{r}, \hat{s})\rangle \mathbf{I}(\vec{r}, \hat{s})+\langle\mathbf{a}(\vec{r},-\hat{s})\rangle B[T(\vec{r})]+\int \mathrm{d} \Omega^{\prime}\left\langle\mathbf{Z}\left(\vec{r}, \hat{s}, \hat{s}^{\prime}\right)\right\rangle \mathbf{I}\left(\vec{r}, \hat{s}^{\prime}\right),
$$

where $\mathbf{I}(\vec{r}, \hat{s})=[I, Q, U, V]^{\mathrm{T}}$ is the four element column vector of radiances (Stokes vector) evaluated at position $\vec{r}$ in direction $\hat{s},\langle\mathbf{K}\rangle$ is the total extinction matrix, $\langle\mathbf{a}\rangle$ is the total absorption vector, $B$ is the Planck function, $\langle\mathbf{Z}\rangle$ is the total phase matrix, $T$ is the temperature of the medium and $\mathrm{d} r$ is a path-length increment. All quantities implicitly depend on the frequency $v$ of the radiation. Eq. (1) can also be expressed in the integral form:

$$
\mathbf{I}\left(\vec{r}_{1}, \hat{s}\right)=\mathcal{O}\left(\vec{r}_{0}, \vec{r}_{1}\right) \mathbf{I}\left(\vec{r}_{0}, \hat{s}\right)+\int_{\vec{r}_{0}}^{\vec{r}_{1}} \mathcal{O}\left(\vec{r}, \vec{r}_{1}\right)[\underbrace{\langle\mathbf{a}(\vec{r},-\hat{s})\rangle B[T(\vec{r})]}_{\text {emission }}+\underbrace{\int \mathrm{d} \Omega^{\prime}\left\langle\mathbf{Z}\left(\vec{r}, \hat{s}, \hat{s}^{\prime}\right)\right\rangle \mathbf{I}\left(\vec{r}, \hat{s}^{\prime}\right)}_{\text {scattering }}] \mathrm{d} r
$$

where $\mathcal{O}\left(\vec{r}_{0}, \vec{r}_{1}\right)$ is the evolution operator from point $\vec{r}_{0}$ to point $\vec{r}_{1}$. For a homogeneous path it is given by

$$
\mathcal{O}\left(\vec{r}_{0}, \vec{r}_{1}\right)=\exp [-\langle\mathbf{K}(\hat{s})\rangle \Delta r]
$$

where $\hat{s}$ is the direction specified by $\vec{r}_{0}-\vec{r}_{1}$, and $\Delta r=\left|\vec{r}_{0}-\vec{r}_{1}\right|$.

Several methods for numerically solving the $V R T E$ for a scattering and emitting atmosphere as required for the $M W$ region, are extensively described in the literature in books like Liou [17]; Chandrasekhar [18]; Thomas and Stamnes [19]; Tsang et al. [1,20]; Lenoble [21]; Goody and Young [22] or in reviews like Haferman [2]; Gasiewski [23]; Mätzler [3]. In this work, we consider three techniques of solving the 3-D VRTE: a backward Monte Carlo, a forward Monte Carlo (with importance sampling), and a discrete ordinate iterative model. A brief description of the three methodologies is provided hereafter together with the essential references.

\subsection{Backward Monte Carlo (ARTS-MC)}

The ARTS Monte Carlo (ARTS-MC) scattering module offers an efficient method for polarized radiative transfer calculations in arbitrarily complex 3-D cloudy cases. The ARTS-MC algorithm is described in detail in Davis et al. [6]. The algorithm solves the integral form of the VRTE, given in Eq. (2), by applying Monte Carlo integration with importance sampling (MCI). The algorithm may be pictured as tracing a large number of photons backwards from sensor, in randomly selected multiply scattered propagation paths to either their point of emission, or entry into the scattering domain. Davis et al. [6] gives a full description of the probability density functions used to determine these propagation paths. This physical picture is identical to the backward-forward Monte Carlo (BFMC) algorithm described by Liu et al. [16]. However, BFMC does not account for dichroism, which is correctly accounted for in ARTS-MC by importance sampling, where every Stokes vector contribution is properly weighted according to its probability density. ARTS-MC also utilizes MCI for convolving the simulated Stokes vector with a 2-D antenna response function, for the detailed simulation of remote sensing observations. 
Being part of the ARTS software package (http://www.sat.uni-bremen.de/arts/), ARTS-MC uses 3-D spherical geometry, which is important for remote sensing applications with large zenith viewing angles, especially limb sounding.

\subsection{Forward Monte Carlo (fMC)}

Battaglia and Mantovani [7] developed a plane parallel polarized code for oriented symmetric particles and extended it to a 3-D fully polarized version, the one exploited in this work. In this code anisotropic effects are taken into account both in the propagation and in the scattering events (not in the propagation segment only like in the procedure followed by Roberti and Kummerow [24]). This avoids the introduction of biasing techniques that can result in unphysical radiation contributions (i.e. with Stokes vector outside of the Poincare sphere). A considerable reduction in computational time is obtained thanks to biasing techniques (extensively reviewed by Roberti [25]). The brightness temperatures are computed at some prescribed viewing angles and averaged over finite regions when 3-D or 2-D problems are considered. As such, the code can actually simulate only problems where spatially averaged quantities are sought (like the radiation sensed by radiometers with finite footprints). With the inclusion of biasing techniques, the computing time problem becomes "manageable" for $M W$ scenarios where total optical thickness does not exceed several units.

\subsection{Discrete ordinate iterative method (DOIT)}

Besides the Monte Carlo module the ARTS model includes the discrete ordinate iterative (DOIT) module for the computation of polarized radiative transfer in cloudy atmospheres [5]. Usually DOIT is only used for 1-D radiative transfer calculations, because the 3-D mode has several difficulties. The DOIT method basically works as follows: the radiation field is discretized in the spatial domain (pressure, latitude, longitude) and in the angular domain (zenith and azimuth angle). Solving the radiative transfer equation for the whole atmosphere would be computationally too expensive, therefore it is solved only for that part of the atmosphere (called the "cloud box") which contains the scattering hydrometeors. As a boundary condition the incoming clear sky radiances are required. The interpolated clear sky field is used as a first guess to compute the scattering integral within the cloud box. Using the first guess scattering integral the radiative transfer equation can be solved at all points in the cloud-box for all possible propagation directions. Thus a new radiation field is obtained which again is used to compute the scattering integral and the radiative transfer equation. These iterations are repeated until convergence is reached. The major difficulty of the method is to find appropriate grids for the accurate representation of the radiation field in both the spatial domain and the angular domain. A grid optimization method is used in order to find the appropriate zenith angle grid for 1-D calculations, but so far there is no grid optimization for the spatial domain and for the azimuth angle grid. Another difficulty related to the computational grids is the CPU time, which increases approximately by a factor of $2^{5}$ if the resolutions of the spatial and angular grids are doubled.

\section{Benchmark result: raining cloud box at $19.4 \mathrm{GHz}$}

We have considered a homogeneous $4 \times 4 \times 4 \mathrm{~km}^{3}$ cloud containing horizontally oriented raindrops, which are modelled as oblate spheroids. The axial ratios (lower than 1) are parameterized according to Andsager et al. [26] as a function of equivalent spherical raindrop diameter $D(\mathrm{in} \mathrm{cm})$ :

$$
\frac{b}{a}=1.0048+0.0057 D-2.628 D^{2}+3.682 D^{3}-1.677 D^{4},
$$

while the drops have an exponential Marshall and Palmer size distribution [27], with a rainfall rate equal to $25 \mathrm{~mm} / \mathrm{h}$. The refractive index of water is assumed to be $5.8393+i 2.9604$ throughout the cloud. The nadir vertical optical thickness of the cloud is 2.33 while the horizontal optical thickness is 2.39 and 1.89 for horizontally and vertically polarized radiation, respectively. The single scattering albedo (direction and polarization dependent) is around 0.25 . The surface is assumed to be a blackbody with temperature $T_{\mathrm{s}}=283.15 \mathrm{~K}$. The model atmosphere is saturated with a linear lapse rate of $-5 \mathrm{~K} / \mathrm{km}$, therefore the 


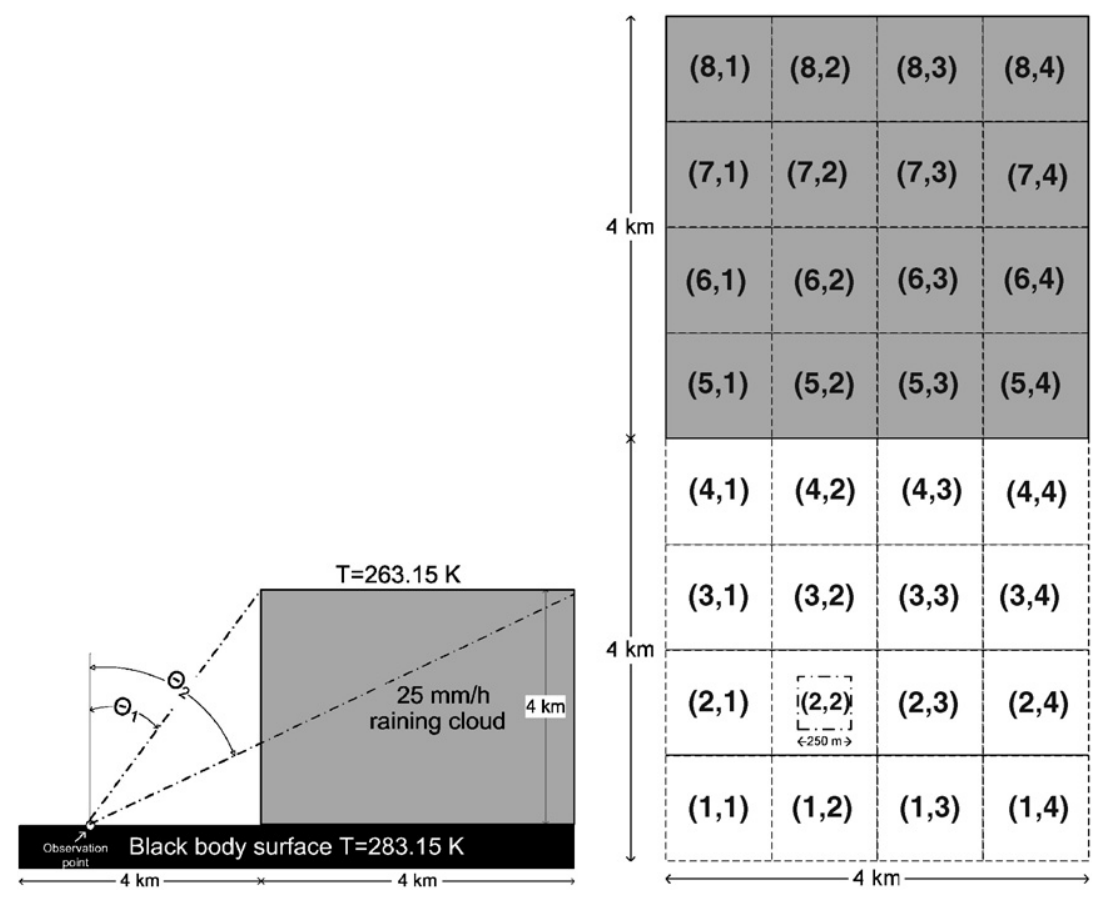

Fig. 1. Schematic for the rain cloud simulation: lateral view (left panel) and top view (right panel). Radiances have been computed at observation points located at the centre of each grid labelled by indices $(i, j)$ with $i=1, \ldots, 8$ and $j=1, \ldots, 4$. The grey shaded area contains the rain system. White areas contain only atmospheric gases.

temperature decreases from 283.15 close to the surface to $263.15 \mathrm{~K}$ at $4 \mathrm{~km}$. Cosmic radiation impinges at $T_{\mathrm{c}}=2.7 \mathrm{~K}$ at the top of the atmosphere. A schematic of the simulation is shown in Fig. 1. This simple scenario was chosen to ensure straightforward implementation in the three RT models, thus reducing the likelihood of differences in model output due to input inconsistency. Also, this scenario follows a previous modelling example by Czekala et al. [28] and can explain important features like the polarization signatures observed at the top and at the bottom of a raining layer. In particular, ground-based observations have confirmed the presence of negative polarization (see [29-31]) in downwelling radiation coming from raining clouds. Example ground-based $Q$ observations at $19.2 \mathrm{GHz}$, and at $30^{\circ}$ elevation angle, are shown in Czekala et al. [30]. $Q$ shows a typical negative signal first decreasing with increasing $T_{\mathrm{B}}$, then saturating around $200 \mathrm{~K}$ and finally increasing towards zero for $T_{\mathrm{B}}>220 \mathrm{~K}$ ). This behaviour of $Q$ can be explained by $1-\mathrm{D}$ raining cloud containing perfectly aligned non-spherical raindrops. However, in this same observational dataset, Czekala et al. [30] noticed the presence of events with very bad agreement between model and observations. In particular strong negative $Q$ signals down to $-9 \mathrm{~K}$ have been reported, which cannot be simulated in a 1-D frame. The coincidentally observed high rain rates suggest a convective precipitation type, which is typically of small horizontal scale. Czekala et al. [30] conclude that 3-D effects are very likely to be present in these situations. Because of this observational context, we focus the intercomparison study on the downwelling radiances.

\subsection{Single scattering properties}

The single scattering properties of the rain layer have been computed by the package PyARTS (http:// www. met.ed.ac.uk/ Cdavis/PyARTS/). This method is based on T-matrix computations, [32]; an analytical orientation averaging of the extinction matrix (according to [33]) is included. The integration of the scattering properties over the size distribution has been performed by a Gauss-Laguerre quadrature scheme. For particle distributions of horizontally aligned raindrops that are oriented randomly in the azimuth the 


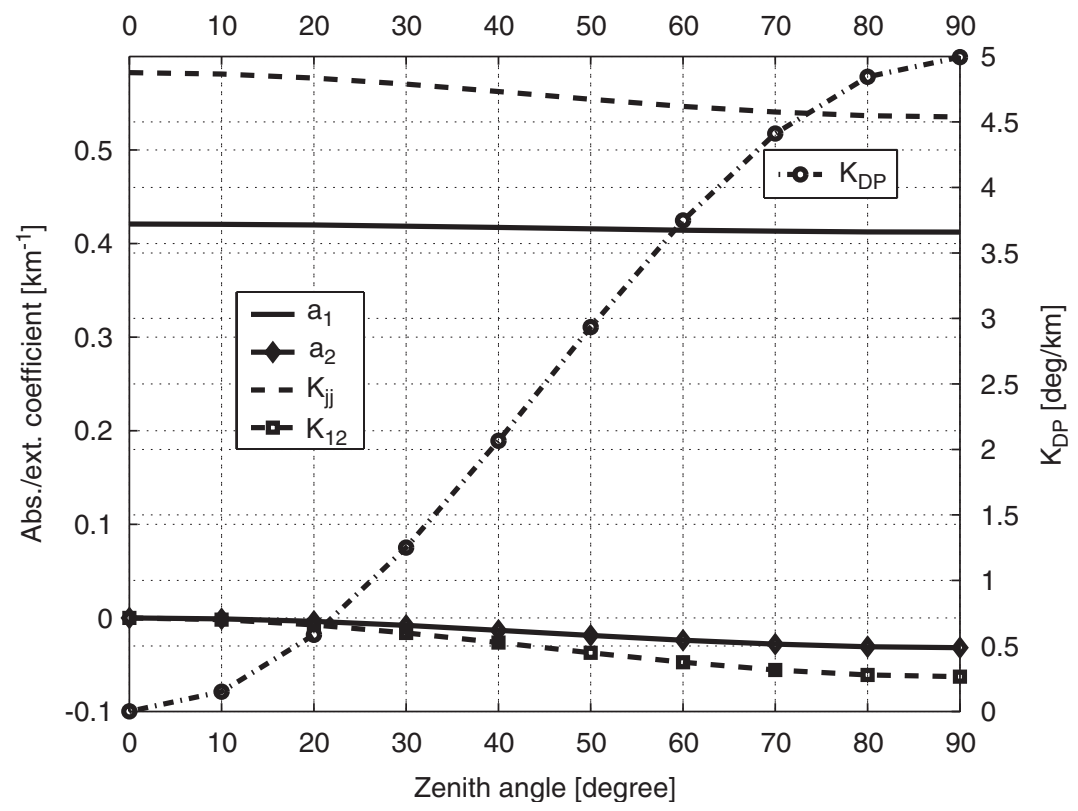

Fig. 2. Extinction matrix elements $K_{j j}$ and $K_{12}$, absorption coefficients $a_{1}$ and $a_{2}$ and $K_{\mathrm{DP}}\left(\propto K_{34}\right)$ for the $R R=25 \mathrm{~mm} / \mathrm{h}$ at $f=19.4 \mathrm{GHz}$.

phase matrix (and also the extinction matrix and the absorption vector) become independent of the incident azimuth angle in this frame. The extinction matrix $\langle\mathbf{K}\rangle$ has only three independent elements [the diagonal term $K_{j j}, K_{12}\left(=K_{21}\right)$, and $\left.K_{34}\left(=-K_{43}\right)\right]$ while the absorption coefficient vector has only the first two elements $a_{1}$ and $a_{2}$ different from zero (Fig. 2). At vertical incidence $K_{12}=a_{2}=0$ while both become negative at zenith angles different from zero: in fact, due to the alignment and the oblateness of the raindrops the absorption/ emission and extinction for the horizontal polarized radiation is larger than for the vertical component. The differential propagation phase $K_{\mathrm{DP}}$, depicted in Fig. 2 (but with the reference $y$-scale on the right-hand side) is identical to $K_{34}$ except for the conversion to degrees $/ \mathrm{km}$.

The phase matrix $Z$ depends on the zenith angle $\theta_{i}$ of the incident radiation, on the zenith angle $\theta_{s}$ of the scattered radiation and on the relative azimuthal angle $\Delta \Phi=\Phi_{i}-\Phi_{s}$. Due to the reciprocity relationship (see chapter 1 in [34]), and to the symmetry of this case, we need only half of the angular grids for $\Delta \Phi$ and half for $\theta_{i}$. The scattering phase matrix elements are available at the web-site http: / /www. meteo. uni-bonn. de/ mitarbeiter/battaglia/.

\subsection{Models setup}

ARTS-MC simulations were done on several $2.8 \mathrm{GHz}$ dual CPU PCs, some with hyper-threading, some without. Up to four simulations were run concurrently on hyper-threaded machines and up to two were run concurrently on machines without hyper-threading. The simulations were run with an accuracy of $0.01 \mathrm{~K}$ for the first Stokes component, but with a time-limit of 2000s. In most cases, this time limit was reached, with the actual accuracy ranging from 0.01 to $0.02 \mathrm{~K}$. With the exception of viewing directions that miss the cloud, the number of photons used was of the order of $10^{7}$. The atmospheric grid had 20 pressure levels, linearly spaced with respect to altitude. Both the latitude and longitude grids incorporated a course grid of 20 points between -4.0 and $+4.0^{\circ}$, merged with a fine grid of 10 points spanning the area of the cloud. The large extent of the coarse grid stems from the requirement that in ARTS all incoming radiation must enter from the top of the atmosphere or the earth surface, not from a side-face.

The $f M C$ grid has a natural Cartesian configuration, so that the rain shaft was positioned at the centre of the $140 \mathrm{~km}$ wide background clear sky atmosphere. The collection grid consists of $64 \times 128$ pixels, each 
$250 \times 250 \mathrm{~m}^{2}$ wide, which cover the whole region drawn in the right panel of Fig. 1 . The radiation field has been computed at 20 zenith angle both at the surface and at the top of the rain layer $(4 \mathrm{~km})$. On a one $1.7 \mathrm{GHz}$ single CPU PC, the simulation took approximately 10 days.

For the ARTS-DOIT simulations, only one $3.06 \mathrm{GHz}$ single CPU PC was used. The spatial grid resolution was exactly the same as for the ARTS-MC simulations. The 1D-optimized zenith angle grid included 74 points and an equidistant grid including 37 points was taken as azimuth angle grid. The convergence limit was $0.1 \mathrm{~K}$ for the first Stokes component and $0.01 \mathrm{~K}$ for the other components. Note that the convergence limit does not correspond to the accuracy of the results, because errors due to the missing grid optimization for 3-D calculations dominate. All results are obtained from a single calculation which took approximately $18 \mathrm{~h}$ computation time.

\section{Results}

The downwelling Stokes vector at the ground is computed at different positions relative to the rain shaft for 21 zenith viewing angles, specified by $\mu=\cos \Theta$, sampled between -1 and 0 with step 0.05 . The radiances are simulated as sensed by a radiometer with an infinitely fine angular resolution either located underneath the cloud [grid points $(i, j)$ with $i=5, \ldots, 8$ in Fig. 1] or looking at the side of the cloud from outside of the rain shaft (grid points with $i=1, \ldots, 4$ in Fig. 1). While the ARTS-MC and the ARTS-DOIT-3D compute the Stokes vector exactly at the centre of each grid box, $f M C$ averages the Stokes vector over a finite horizontal region of $250 \mathrm{~m} \times 250 \mathrm{~m}$. This region is indicated by a dash-dotted square only for the grid $(2,2)$ in Fig. 1 . To assess the variability of the radiances inside this region the results of the radiances computed by averaging over this small region are evaluated with another backward scheme (see discussion in Section 4.3).

\subsection{I and $Q$ channels}

Results for $I$ and $Q$ at grid points with $j=1$ (see right panel of Fig. 1) are plotted in Fig. 3: the upper four panels refer to observation points outside of the cloud, the last four panels to observations from underneath the cloud. Let us consider the $I$ and $Q$ values as computed at the centre of pixel $(1,1)$ (top panels in Fig. 3). As inferable from the sketch in the left panel of Fig. 1 the radiance field is not affected by the raining cloud until the zenith angle becomes greater than $\Theta_{1}=41.2^{\circ}(\mu=0.75)$. Therefore in the interval between $\mu=1$ and 0.75 the intensity corresponds to clear sky radiance and there is no polarization. For zenith angles greater than $\Theta_{1}$ the polarization signal has a sudden steep decrease down to values lower than $-4.5 \mathrm{~K}$. This is due to the fact that at small slant optical thickness both the emitted and the scattered contributions to polarization tend to be negative. For zenith angles greater than $53.1^{\circ}$ the polarization increases again; here the optical thickness becomes considerably higher than 1 . A relative maximum is then found at $\Theta_{2}=61.9^{\circ}$, i.e. $\mu=\cos \left(\Theta_{2}\right)=0.47$. At this angle the slant geometrical thickness $r_{\mathrm{sl}}$ through the cloud is $4.5 \mathrm{~km}$. This corresponds to a slant optical thickness equal 2.6 for unpolarized radiation. With further increasing zenith angles another minimum is found around $\mu=0.25$; then $Q$ goes back to values close to zero or even slightly positive at grazing angles. The intensity has a much smoother behaviour with a constant increase in $T_{\mathrm{B}}$ when moving toward higher zenith angles and the well-known saturation of $T_{\mathrm{B}}$ at large optical thicknesses.

When approaching the cloud with the observation point (second line panels in Fig. 3) the transition between clear sky and rain is practically absent. Since the observation point $(4,1)$ is $0.5 \mathrm{~km}$ from the cloud edge, the transition occurs at $\mu=0.9923$ while the first computed point different from the zenith direction is at $\mu=0.95$. The $T_{\mathrm{B}} \mathrm{s}$ immediately adjust to warmer values, characteristic of high cloud optical thickness. Now $Q$ decreases not as fast as before and the hump structure practically disappears (except for the inflection point at $\mu=0.66$ ). For zenith angles with $\mu \leq 0.25$ the behaviour of both $I$ and $Q$ is quite similar to the one recorded at position $(1,1)$. This is expected since the radiometer is sensing in both cases the full horizontal extent of the rain shaft.

When observing the rain shaft from underneath the cloud at position $(5,1)$ a cusp is observed in the intensity pattern. In this configuration the observed optical thickness of the cloud increases when scanning from nadir to $41.2^{\circ}(\mu=0.75)$, correspondingly also $T_{\mathrm{B}}$ increases. The successive decrease is due to the reduction of the sensed slant optical thickness. At grazing angles the optical thickness increases again due to increasing path within the pure gas absorption region. When going further inside the cloud $(i=6,7)$ the position of the 

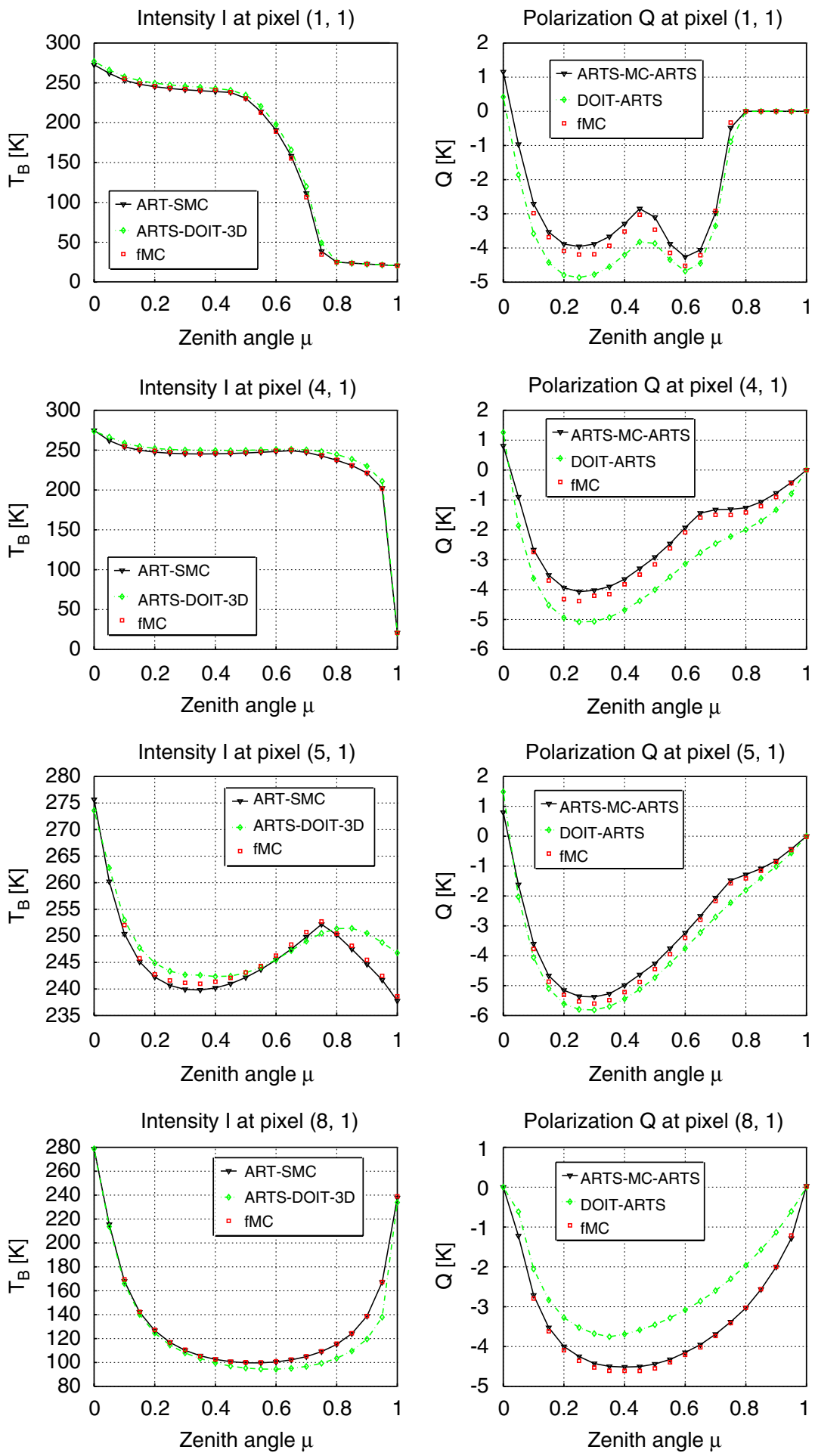

Polarization $Q$ at pixel $(8,1)$

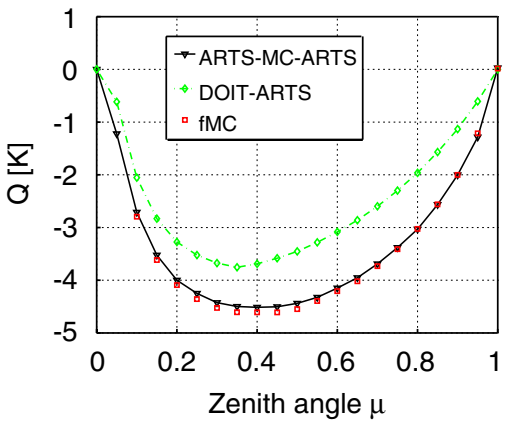

Fig. 3. Intensity $I$ (left panel) and polarization difference $Q$ (right panel) evaluated at different grid points as indicated in the title of each panel (use Fig. 1, right panel, as a reference). 
maximum moves closer to nadir. At position $(8,1)$ the maximum is so close to nadir that it cannot be captured by our zenith angle sampling. In this case $T_{\mathrm{B}}$ is much colder than in the former case because of the reduced cloud optical thickness. $Q$ has a typical concave shape for all positions underneath the cloud with the position of the minimum being around $\mu=0.3,0.4$ and minimum values of polarization reaching around -6 and $-4.5 \mathrm{~K}$ for position $(5,1)$ and $(8,1)$, respectively. Note that for position $(7,2)$ (not shown) $Q$ can go down to $-8 \mathrm{~K}$. In a $1-\mathrm{D}$ rain shaft with the same vertical structure $Q$ never reaches values lower than $-4 \mathrm{~K}$ at all viewing angles. Thus these findings credit the hypothesis of 3-D effects formulated by Czekala et al. [30] as possible explanation for the ground-based observations of strongly negative $Q$ (for further details see [35]).

Pure geometrical considerations allow the conclusion that the same $Q$ patterns are symmetric with respect to the line $x=2 \mathrm{~km}$ in the right-hand panel of Fig. 1, e.g. $Q(5,2)=Q(5,3)$. Note that, because of the finiteness of the cloud, for some pixels, $Q$ values different from zero are found even at nadir. This is due to the asymmetry between orthogonal horizontal directions introduced by the finiteness of the cloud. The geometric structure of the cloud implies that $Q_{\text {nadir }}(5,1)=Q_{\text {nadir }}(6,2)=0$ while $Q_{\text {nadir }}(5,2)=-Q_{\text {nadir }}(6,1) \neq 0$. The two different Monte Carlo codes are capturing these features. For instance $Q_{\text {nadir }}(6,1)$ assumes values between -0.55 and $-0.75 \mathrm{~K}$. On the other hand ARTS-DOIT-3D always gives zeros values for $Q$ at nadir.

\section{2. $U$ and $V$ channels}

The presence of a 3-D structures represents the source of non-zero $U$ and $V$ values as well. For these parameters we expect an anti-symmetry with respect to the line $x=2 \mathrm{~km}$, i.e. $U(:, k)=-U(:, 4-k)$ and $V(:, k)=-V(:, 4-k)$ with $k=1,2$. $U$ and $V$ are shown in Fig. 4 for the same pixels considered in Fig. 3. Generally, the $U$ is an order of magnitude smaller than the $Q$; however, it can reach values higher than $1 \mathrm{~K}$. ARTS-DOIT-3D always gives values close to zero; obviously this model is not able to simulate such small polarization difference values. On the other hand very good agreement is found between ARTS-MC and $f M C$ simulations. Similarly to the $Q$ values, also when looking at $U_{\text {nadir }}$ Monte Carlo results respect the symmetry $U_{\text {nadir }}\left(6, \frac{1}{2}\right)=-U_{\text {nadir }}\left(7, \frac{1}{2}\right) \neq 0$ and $U_{\text {nadir }}\left(5, \frac{1}{2}\right)=-U_{\text {nadir }}\left(8, \frac{1}{2}\right) \neq 0$ with opposite values when $U$ is evaluated in grids the column of which is labelled with index $j$ equal to 3 or 4.

$V$ is an order of magnitude smaller than $U$, so that this channel becomes even more sensitive to different $R T$ models. In this case also ARTS-MC and $f M C$ substantially disagree. However, the coupling of $V$ is that weak that it does not substantially affect the other Stokes parameters.

\subsection{Relative accuracy of the codes}

Relative errors between the three different codes in correspondence to the panels shown in Fig. 3 are depicted in Fig. 5. The Monte Carlo codes can provide an estimate of the statistical error based on the variance of the Stokes component derived from the prescribed number of photons. Generally, backward Monte Carlo schemes have negligible statistical errors, because it is computationally feasible to run enough photons to reduce the typical vertical and horizontal $T_{\mathrm{B}}$ errors to less than $0.01 \mathrm{~K}$. The statistical error of the $f M C$, however is still not negligible (see the error bars in Fig. 5) because of the high computational cost of the procedure. In Fig. 5 all variables are compared to the results obtained by the ARTS-MC scheme, which is taken as a reference. The ARTS-DOIT-3D results are biased with respect to the other two codes especially when looking at the cloud from the inside [e.g. differences up to $30 \mathrm{~K}$ in $T_{\mathrm{B}}$ and up to $1.1 \mathrm{~K}$ in $Q$ can be found at pixel $(8,1)$, bottom panels in Fig. 5]. There is a good agreement between the ARTS-MC and the $f M C$, particularly for points underneath the cloud $(\Delta Q \leq 0.2 \mathrm{~K}$ and $\Delta I \leq 1 \mathrm{~K})$. Large differences (as large as $5 \mathrm{~K}$ in $I$ and $0.4 \mathrm{~K}$ in $Q$ ) are found when looking at the rain shaft from the outside. Regarding $U$ and $V$, only an intercomparison between the different Monte Carlo procedures is performed (ARTS-DOIT-3D always gives $U$ and $V$ close to zero). For $U$ the difference between $f M C$ and ARTS-MC are never higher than $0.1 \mathrm{~K}$ and the general structure of the signal is similar. But $V$ results in completely different patterns. In both cases the signal remains, however lower than $0.25 \mathrm{~K}$.

To explain the small differences between ARTS-MC and $f M C$ we have to stress that while ARTS-MC and ARTS-DOIT-3D are part of the same software package, $f M C$ is a separate code, with its own $I / O$ routines. The reason for the disagreement between ARTS-MC and $f M C$ may depend on different tracing and scattering 

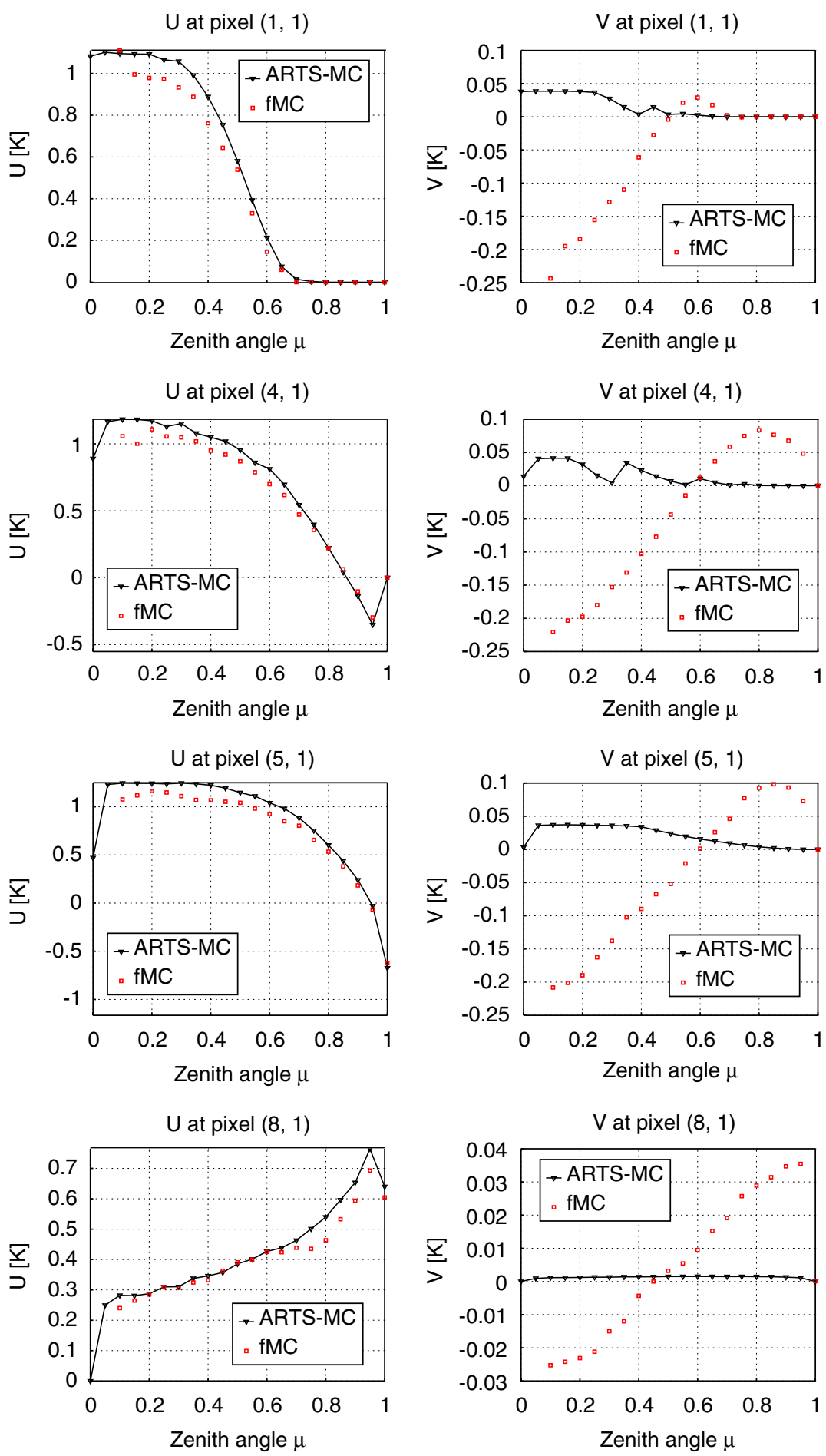

Fig. 4. $U$ (left panels) and $V$ channel (right panels) evaluated at different grid points as indicated in the title of each panel (use Fig. 1, right panel, as a reference).

procedures and in particular different interpolation schemes when dealing with the phase function. A large part of the discrepancy could be due to the difference between spherical (ARTS-MC), and Cartesian (fMC) geometry. This difference will result in significant differences in incoming radiation at zenith angles close to the 
Relative errors in $T_{B}$ at pixel $(1,1)$

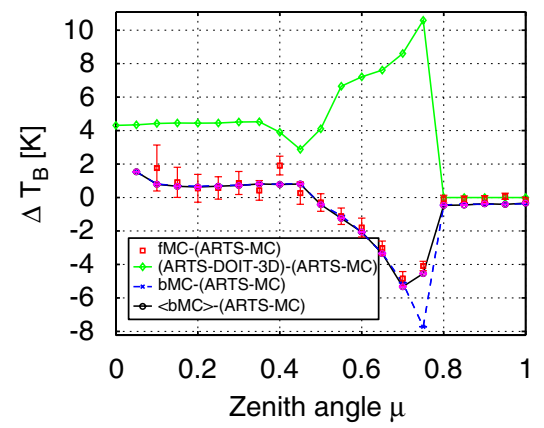

Relative errors in $\mathrm{T}_{\mathrm{B}}$ at pixel $(4,1)$

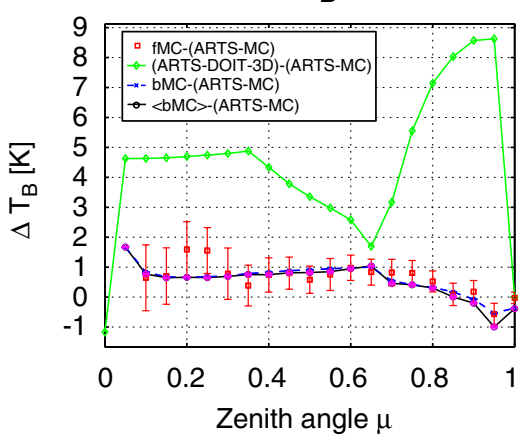

Relative errors in $\mathrm{T}_{\mathrm{B}}$ at pixel $(5,1)$

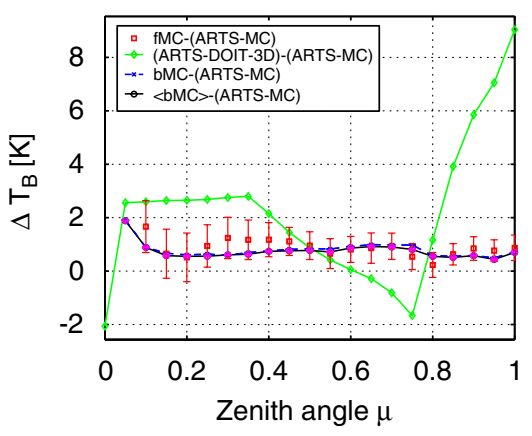

Relative errors in $\mathrm{T}_{\mathrm{B}}$ at pixel $(8,1)$

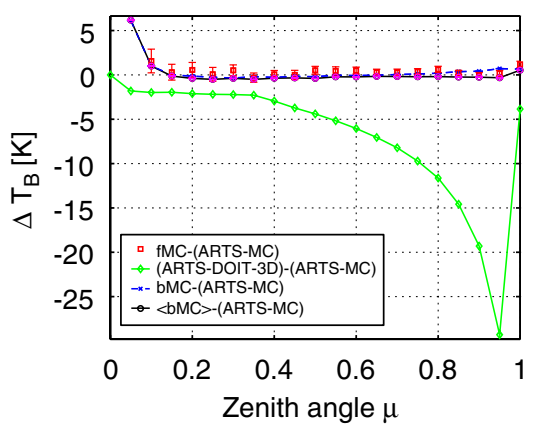

Relative errors in $Q$ at pixel $(1,1)$

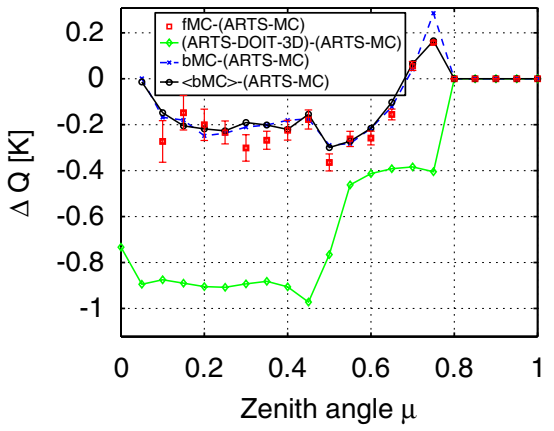

Relative errors in $Q$ at pixel $(4,1)$

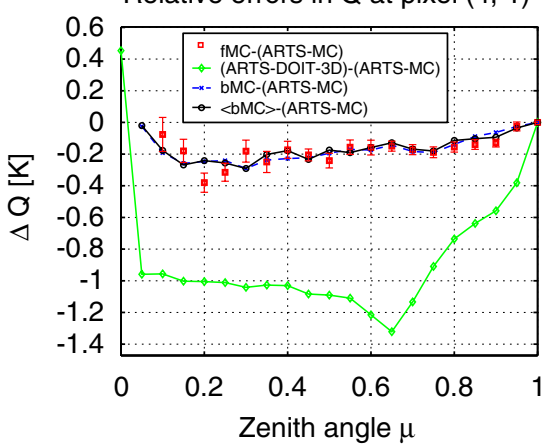

Relative errors in $Q$ at pixel $(5,1)$
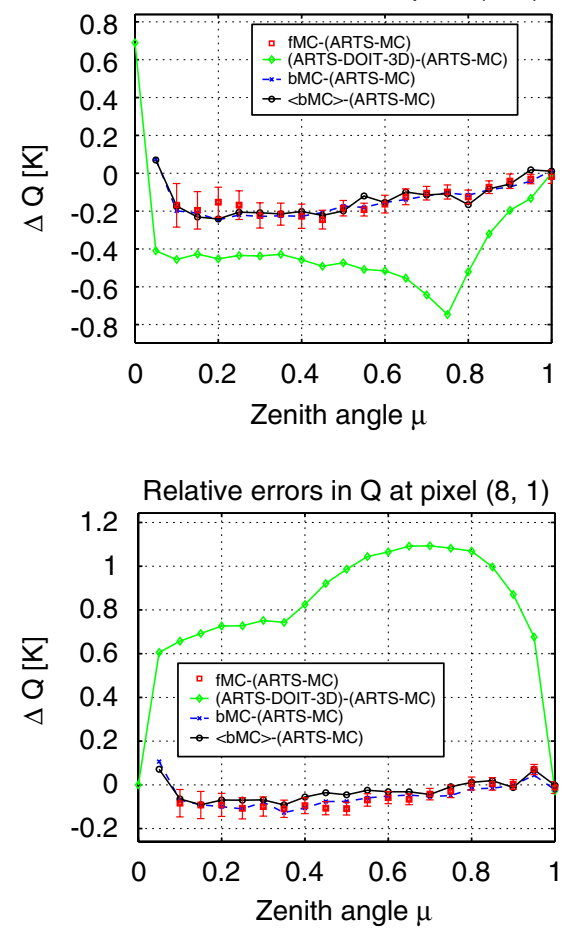

Fig. 5. Relative errors of ARTS-DOIT-3D, $f M C, b M C$ and $\langle b M C\rangle$ in correspondence to panels in Fig. 3. ARTS-MC is taken as a reference. 
limb, which will affect the scattering integral calculation. Aside from the difference in geometry, details of atmospheric grid discretization and interpolation will also lead to small differences.

To verify that the cause of the disagreement resides in implementation issues such as those mentioned above, and not in some fundamental problem with either ARTS-MC or $f M C$, the backward methodology described in Davis et al. [6] was implemented in the same software framework as $f M C$ so that both algorithms could be compared within Cartesian geometry, and with the same $I / O$ and sampling routines. We refer to this new implementation as $b M C$ (and this is now also available at http://www.meteo.uni-bonn.de/mitarbeiter/ battaglia/).

In the panels of Fig. 5 we have included results from the $b M C$ in two configuration: one which computes the radiation at the centre of the grids (like ARTS-MC and ARTS-DOIT-3D) and another one which averages the radiation field over the averaging area of the $f M C$ scheme (thus the abbreviation $\langle b M C\rangle$ ). Note that the $\langle b M C\rangle$ and the $b M C$ are always very close: this means that the radiation field is almost linear in the averaging region. Only in the upper left panel of Fig. 5 there are consistent biases between the two codes in terms of intensities with values as high as $4 \mathrm{~K}$ for viewing angles $\mu$ around 0.75 . This is expected because these angles correspond exactly to the cloud edge; in the sharp transition between pure sky and precipitating cloud the radiation field for the same viewing angle is expected to have strong gradients, especially when moving in the $y$-direction (see Fig. 1). Therefore, the differences between the ARTS-MC and the $f M C$ cannot be attributed to the spatial averaging performed by the $f M C$. For $I, Q, U$, and $V$ the differences between $b M C$ and $f M C$ were less than than $1,0.1,0.1$ and $0.02 \mathrm{~K}$, i.e. lower than the statistical error $\mathrm{d} I, \mathrm{~d} Q, \mathrm{~d} U, \mathrm{~d} V$, respectively. This confirms that the small differences between ARTS-MC and $f M C$ were due to the implementation differences outlined above, and that the backward and forward Monte Carlo methods are indeed consistent.

\section{Conclusions and recommendations}

Radiative transfer computations with a 3-D scenario including a rain shaft with perfectly oriented raindrops have been performed with three different methodologies, a backward Monte Carlo, a forward Monte Carlo and a discrete ordinate iterative method. Although quite simple the scenario is believed to be an interesting test-bed because it can be related to ground-based observations and because all four Stokes vector are different from zero. Results demonstrate a qualitative agreement between all methods for the first two Stokes components. However, a quantitative agreement has been found only among the Monte Carlo codes whereas the ARTS-DOIT-3D methodology agrees only qualitatively with the Monte Carlo outputs. The reason for that has to be ascribed to the difficulty of ARTS-DOIT-3D to describe a radiation field with strong gradients like that generated by the sharp boundaries of the raining shaft. For this reason 3D DOIT simulations can only be used in order to estimate the cloud effect qualitatively. On the other hand ARTS-MC and $f M C$ generally agree well in all channels with the only exception of $V$ (that is any way always smaller than $0.3 \mathrm{~K}$ ). An even better agreement has been found between $f M C$ and $b M C$ (especially in the run $\langle b M C\rangle$ ), both coded within the same $f 77$ platform (with common subroutines for tracing and scattering) and the same $I / O$ frame, and with a Cartesian grid. This demonstrates the equivalence between the forward methodology (see [7]) and the backward-forward methodology (see [6]). The remaining disagreement between ARTS-MC and $f M C$ is believed to be attributable to differences in the atmospheric grid set-up (spherical in the ARTS-MC) and in the tracing and scattering procedures. The $f M C$, as implemented, has very high computational costs (in our simulation it is more than one order of magnitude slower than the $b M C$, still with some not negligible statistical noise!).

When compared to other techniques for solving the VRTE the ARTS-MC algorithm has some advantages:

- All computational effort is dedicated to calculating the Stokes vector at the location of interest and in the direction of interest. This is particularly relevant for space-borne remote sensing, where we are only interested in a narrow field of view. This is in contrast to forward Monte Carlo and discrete ordinate methods where the whole radiation field is calculated.

- CPU and memory cost scales more slowly with grid size than discrete ordinate methods. Thus large or detailed 3-D scenarios are not a problem. This stems from the suitability of MCI for evaluating integrals over highly dimensioned spaces. In addition to the CPU cost, which increases dramatically in 3D DOM 
applications with the number of grid-points in each dimension, also the memory requirements become prohibitive at moderate grid sizes due to the requirement that the radiance in every direction must be stored at every grid point.

- Optically thick media are no problem. A feature of backward Monte Carlo algorithms is that only parts of the atmosphere that actually contribute to the observed radiance are considered in the computation. So where the medium is optically thick due to absorption or scattering, only the parts of the atmosphere closest to the sensor are "visited" by the algorithm. This contrasts with DOM and $f M C$ methods, which require the computation of the whole radiation field and where added optical thickness further restricts the number of photons reaching the sensor.

This study confirms that the backward Monte Carlo technique based on importance sampling represents currently the most efficient way to face polarized radiative transfer problems within thick 3-D-structured clouds including non spherical preferentially oriented hydrometeors. It should be noted that this recommendation applies only for passive remote sensing applications and long wavelengths, where strong localized sources can be neglected.

\section{References}

[1] Tsang L, Kong JA, Ding K. Scattering of electromagnetic waves: theories and applications. New York: Wiley-Interscience; 2000.

[2] Haferman JL. In: Mishchenko MI, Hovenier JW, Travis LD. editors, Light scattering by nonspherical particles: theory, measurements, and applications, chapter Microwave scattering by precipitation. San Diego: Academic Press; 2000.

[3] Mätzler C, Thermal microwave radiation - applications for remote sensing, IET Electromagnetic Waves Series. London, UK; 2006.

[4] Haferman JL, Krajewski WF, Smith TF, Sanchez A. Radiative transfer for a three-dimensional raining cloud. Appl Opt 1993;32: 2795-802.

[5] Emde C, Buehler SA, Davis C, Eriksson P, Sreerekha TR, Teichmann C. A polarized discrete ordinate scattering model for simulations of limb and nadir longwave measurements in 1D/3D spherical atmospheres. J Geophys Res 2004;109(D24).

[6] Davis CP, Emde C, Harwood RS. A 3D polarized reversed Monte Carlo radiative transfer model for mm and sub-mm passive remote sensing in cloudy atmospheres. IEEE Trans Geosci Remote Sens 2005;43:1096-101.

[7] Battaglia A, Mantovani S. Forward Monte Carlo computations of fully polarized microwave radiation in nonisotropic media. JQSRT 2005;95(3):285-308.

[8] Battaglia A, Prodi F, Porcu F, Shin D-B. In: Levizzani V, Bauer P, Turk FJ. editors, 3D effects in MW radiative transport inside precipitating clouds: modeling and applications. Measuring precipitation from space: EURAINSAT and the future, Dordrecht: Kluwer Academic edition; 2005.

[9] Evans KF, Stephens GL. A new polarized atmospheric radiative transfer model. JQSRT 1991;46:413-23.

[10] Kuik F, Haan JFD, Hovenier QW. Benchmark results for single scattering by spheroids. JQSRT 1992;47:477-89.

[11] Haferman JL, A polarized multi-dimensional discrete-ordinates radiative transfer model for remote sensing applications. PhD thesis, University of Iowa; 1995.

[12] Wauben WM, Hovenier JW. Polarized radiation of an atmosphere containing randomly-oriented spheroids. JQSRT 1992;47: 491-504.

[13] Haferman JL, Smith TF, Krajewski WF. A multi-dimensional discrete ordinates method for polarized radiative transfer. i. Validation for randomly oriented axisymmetric particles. JQSRT 1997;58:379-98.

[14] Evans KF. The spherical harmonics discrete ordinate method for three-dimensional atmospheric radiative transfer. J Atmos Sci 1998; 55:429-46.

[15] Roberti L, Haferman J, Kummerow C. Microwave radiative transfer through horizontally inhomogeneous precipitating clouds. J Geophys Res 1994;99(16):707-16.

[16] Liu Q, Simmer C, Ruprecht E. Three-dimensional radiative transfer effects of clouds in the microwave spectral range. J Geophys Res 1996;101(D2):4289-98.

[17] Liou KN. An introduction to atmospheric radiation, second ed. New York: Academic Press; 2002.

[18] Chandrasekhar S. Radiative transfer. New York: Dover Publications; 1960.

[19] Thomas GE, Stamnes K. Radiative transfer in the atmosphere and ocean. Cambridge: Cambridge University Press; 1999.

[20] Tsang L, Kong JA, Shin RT, Theory of microwave remote sensing. New York: Wiley-Interscience; 1985. ISBN 0-471-88860-5.

[21] Lenoble JE. Radiative transfer in scattering and absorbing atmospheres: standard computational procedures. Hampton, VA: Deepak; 1985.

[22] Goody RM, Young YL. Atmospheric radiation: theoretical basis. New York: Oxford University Press; 1989.

[23] Gasiewski A, Microwave radiative transfer in hydrometeors. Atmospheric remote sensing by microwave radiometry. New York: Wiley; 1993.

[24] Roberti L, Kummerow C. Monte Carlo calculations of polarized microwave radiation emerging from cloud structures. J Geophys Res 1999;104:2093-104. 
[25] Roberti L. Monte Carlo radiative transfer in the microwave and in the visible: biasing techniques. Appl Opt 1997;36(30):7929-38.

[26] Andsager K, Beard KV, Laird NS. A laboratory study of oscillations and axis ratios for large raindrops. J Atmos Sci 1999;56: 2673-83.

[27] Marshall JS, Palmer WM. The distribution of raindrop with size. J Meteorol 1948;5:165-6.

[28] Czekala H, Havemann S, Schmidt K, Rother T, Simmer C. Comparison of microwave radiative transfer calculations obtained with three different approximations of hydrometeor shape. JQSRT 1999;63:545-58.

[29] Kutuza BG, Zagorin GK, Hornbostel A, Schroth A. Physical modeling of passive polarimetric microwave observations of the atmosphere with respect to the third stokes parameter. Radio Sci 1998;33(3):677-96.

[30] Czekala H, Crewell S, Hornbostel A, Schroth A, Simmer C, Thiele A. Interpretation of polarization features in ground-based microwave observations as caused by horizontally aligned oblate rain drops. J Appl Meteorol 2001;40:1918-32.

[31] Troitsky AV, Osharin AM, Korolev AV, Strapp JW. Polarization of thermal microwave atmospheric radiation due to scattering by ice particles in clouds. J Atmos Sci 2003;60:1608-20.

[32] Mishchenko MI. Calculation of the amplitude matrix for a nonspherical particle in a fixed orientation. Appl Opt 2000;39(6):1026-31.

[33] Mishchenko MI. Extinction and polarization of transmitted light by partially aligned nonspherical grains. Astrophys J 1991;367: 561-74.

[34] Mishchenko MI, Travis LD, Lacis AA. Scattering, absorption and emission of light by small particles. Cambridge: Cambridge University Press; 2002.

[35] Battaglia A, Simmer C. Explaining the polarization signal from rain dichroic media, JQSRT, 2006, under revision. 